\title{
Iron Catalyzed Formal [3+2] Cycloaddition of Tetrahydroisoquinoline Affording Amide-bearing Dihydropyrrolo[2,1-a]isoquinolines
}

Si-Wei Liu, Dan-Dan Ma, Xin-Xin Zhu, Cheng-Dan Luo, Hui-Lin Tan, Xiao-Li Ju, Xue Tan, Xiao-Hui Tang, Jie Huang, Jia Wang, Xian-Xun Wang, Hai-Lei Cui

Submitted date: $21 / 03 / 2020$ - Posted date: 25/03/2020

Licence: CC BY-NC 4.0

Citation information: Liu, Si-Wei; Ma, Dan-Dan; Zhu, Xin-Xin; Luo, Cheng-Dan; Tan, Hui-Lin; Ju, Xiao-Li; et al. (2020): Iron Catalyzed Formal [3+2] Cycloaddition of Tetrahydroisoquinoline Affording Amide-bearing Dihydropyrrolo[2,1-a]isoquinolines. ChemRxiv. Preprint. https://doi.org/10.26434/chemrxiv.12015651.v1

We have developed an iron(III) chloride hexahydrate catalyzed formal [3+2] cycloaddition of tetrahydroisoquinolines with amide group-bearing Morita-Baylis-Hillman (MBH) carbonates. A range of highly functionalized dihydropyrrolo[2,1-a]isoquinolines could be prepared through SN2'/oxidation/ electrocyclization/aromatization cascade (32-62\% yield).

File list (2) 


\title{
Iron Catalyzed Formal [3+2] Cycloaddition of Tetrahydroisoquinoline Affording Amide-bearing Dihydropyrrolo[2,1-a]isoquinolines
}

Si-Wei Liu, ,a,b] Dan-Dan Ma,[a] Xin-Xin Zhu,[a] Cheng-Dan Luo,,[a] Hui-Lin Tan, ${ }^{[a] ~ X i a o-L i ~ J u,[a] ~ X u e ~}$

Tan,[a] Xiao-Hui Tang, ${ }^{[a]}$ Jie Huang,[a] Jia Wang, [a] Xian-Xun Wang[b] and Hai-Lei Cui*,[a]

[a] S.-W. Liu , D.-D. Ma, X.-X. Zhu, C.-D. Luo, H.-L. Tan, X.-L. Ju, X. Tan, X.-H. Tang, J. Huang, J. Wang, H.-L. Cui

Laboratory of Asymmetric Synthesis, Chongqing University of Arts and Sciences, 319 Honghe Ave., Yongchuan, Chongqing, 402160, P.R. China

[b] S.-W. Liu, X.-X. Wang

Tonichem Pharmaceutical Technology Co., Ltd, Huizhou, 516008, P.R. China

Corresponding author: cuihailei616@163.com

\begin{abstract}
We have developed an iron(III) chloride hexahydrate catalyzed formal $[3+2]$ cycloaddition of tetrahydroisoquinolines with amide group-bearing Morita-Baylis-Hillman (MBH) carbonates. A range of highly functionalized dihydropyrrolo[2,1-a]isoquinolines could be prepared through SN2'/oxidation/ electrocyclization/aromatization cascade (32-62\% yield).
\end{abstract}




\section{Introduction}

Given the wide occurrence of pyrrolo[2,1-a]isoquinoline in many natural products and bioactive synthetic molecules of great importance as core structure (Figure 1), the construction of pyrroloisoquinoline derivatives has attracted much attention in the field of synthetic chemistry and medicinal chemistry. ${ }^{[1]} \mathrm{A}$ large range of elegant methodologies has been developed, including multistep synthesis ${ }^{[2]}$, 1,3-dipolar cycloaddition ${ }^{[3]}$, multicomponent reaction ${ }^{[4]}$, photoredox catalysis-based reactions ${ }^{[5]}$, electrocyclization ${ }^{[6]}$, and other protocols.[7] Apparently, construction of pyrroloisoquinolines in a direct way could avoid the multistep manipulation, cost of time, chemical waste and labor waste. Thus, from the standpoint of green chemistry and sustainable chemistry, direct synthesis of pyrroloisoquinoline derivatives using readily accessible starting material through one-pot or domino reaction pathway is much desirable.

We have been focusing on the development of highly functionalized azacycle construction by the use of cyclic nucleophilic imines through the formation of 1,n-dipole as the key intermediate (Scheme 1).[8] By this strategy, we have successfully prepared several structurally complex azacycles possessing privileged frameworks, such as dihydropyrroloisoquinolines, pyrroloisoquinolines, pyrroloquinolines, dimeric dihydro- $\beta$-carbolines and dimeric dihydropyrroloisoquinolines. ${ }^{[8]}$ To further simplify the synthetic protocol by the design of cascade reactions, we have therefore realized an iron chloride catalyzed synthesis of dihydropyrroloisoquinoline with dihydroisoquinoline imines and Morita-Baylis-Hillman (MBH) carbonates recently. ${ }^{[9]}$ Along this line, it is reasonable to conclude that the generation of 1,5-dipole could be realized by the oxidation of in situ generated allylic tertiary amine in the presence of iron salts and then the desired azacycles could be obtained through further electrocyclization. ${ }^{[10]}$ In this design, cyclic secondary amines can be used instead of nucleophilic imines to react with electrophiles to produce 1,5-dipoles. The replacement of dihydroisoquinoline imines with tetrahydroisoquinoline amines in this reaction avoids the preparation of imines.
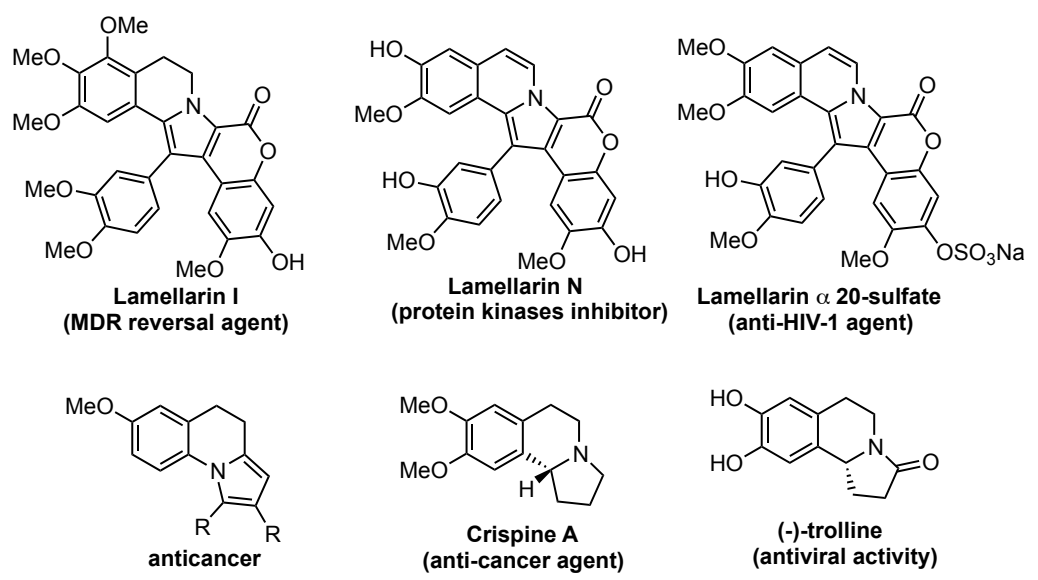

Figure 1. Important Pyrroloisoquinoline Derivatives. 
Previously, Basavaiah and co-workers have developed an impressive $\mathrm{CuBr} / \mathrm{t}$-butyl hydroperoxide promoted synthesis of dihydropyrrolo[2,1-a] isoquinolines and dihydroindolo[2,3-a]indolizines.[11] However, too large amount of metal salts was needed in this process. To avoid the use of stoichiometric amount of metal salts (1 equivalent of copper salts in their study), a catalytic version of reaction system would be more attractive. On the basis of our previous work and inspired by recent success from others, we envisioned that iron salts can be used as catalyst in a designed SN2'/oxidation/electrocyclization/aromatization cascade under air or dioxygen atmosphere. ${ }^{[12]}$ Dioxygen would act as the terminal oxidant for the oxidation of tertiary amine intermediate to 1,n-dipolar and the final aromatization.

In addition, in order to further expand the molecular complexity, the variation of $\mathrm{MBH}$ carbonates would be attractive. ${ }^{[13]}$ There are relatively less reports on the transformations of amide-bearing $\mathrm{MBH}$ adducts. Particularly, the direct use of MBH carbonates bearing amide moiety is less explored. In our previous work, we have successfully realized the incorporation of amide group into the dihydropyrrolo[2,1-a]isoquinolines by the use of preformed $\mathrm{MBH}$ carbonates bearing amide moiety (only one example). Beside of potential change of reactivity of $\mathrm{MBH}$ carbonates in certain reactions, the introduction of amide group may also be beneficial for further modification and transformation of the dihydropyrrolo[2,1-a]isoquinoline derivatives. Here, we report our development of iron(III) chloride hexahydrate catalyzed synthesis of dihydropyrrolo[2,1-a]isoquinolines with tetrahydroisoquinolines and MBH carbonates bearing amide groups.

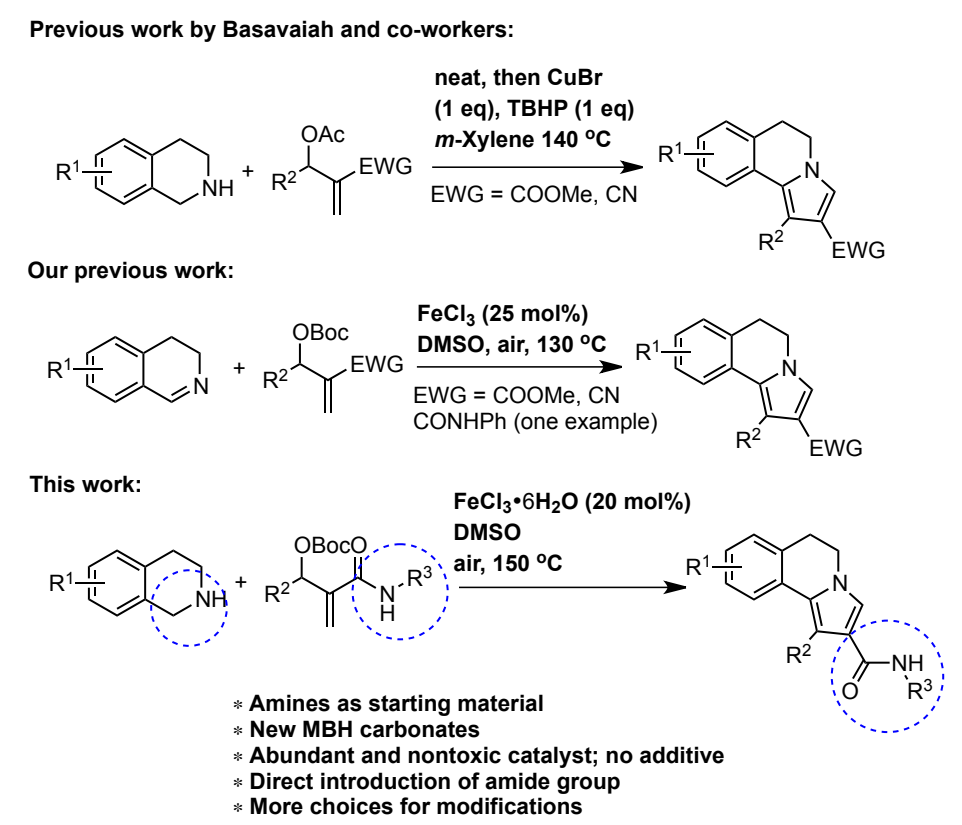

Scheme 1. Synthesis of Dihydropyrrolo[2,1-a]isoquinolines with MBH Derivatives. 


\section{Results and Discussion}

As amide is frequently occurred subunit in natural products, pharmaceuticals, and polymers, the development of methodologies for amide formation has gained growing attention. ${ }^{[14]}$ The synthesis of preformed amide in substrates for cycloaddition is a useful way for introducing amide groups into complex molecules. We hypothesized that the introduction of amide group into the dihydropyrrolo[2,1-a]isoquinolines could be realized by the the utilization of amide-bearing $\mathrm{MBH}$ carbonates. Therefore, the synthesis of novel MBH carbonates has been designed as shown in Scheme 2 inspired by the pioneering studies from the groups of $\mathrm{Lu}$ and Kim on the synthesis of MBH derivatives.[15] We prepared new amide-containing $\mathrm{MBH}$ carbonates through hydrolysis of $\mathrm{MBH}$ adducts, amidation and final carbonation with $\mathrm{Boc}_{2} \mathrm{O}$. As shown in Scheme 2, MBH carbonates could be prepared in 19-68\% yields (total yields for three steps). Both primary and secondary amines can be used as amine source. Pleasingly, thiophene moiety can be incorporated into the MBH carbonate albeit with relatively low yield (compound $\mathbf{2 e}$, total 19\% yield for three steps). This synthetic route could provide easy access to $\mathrm{MBH}$ carbonates bearing various functionalized arylated and alkylated amines derived amides from easily accessible MBH adducts.

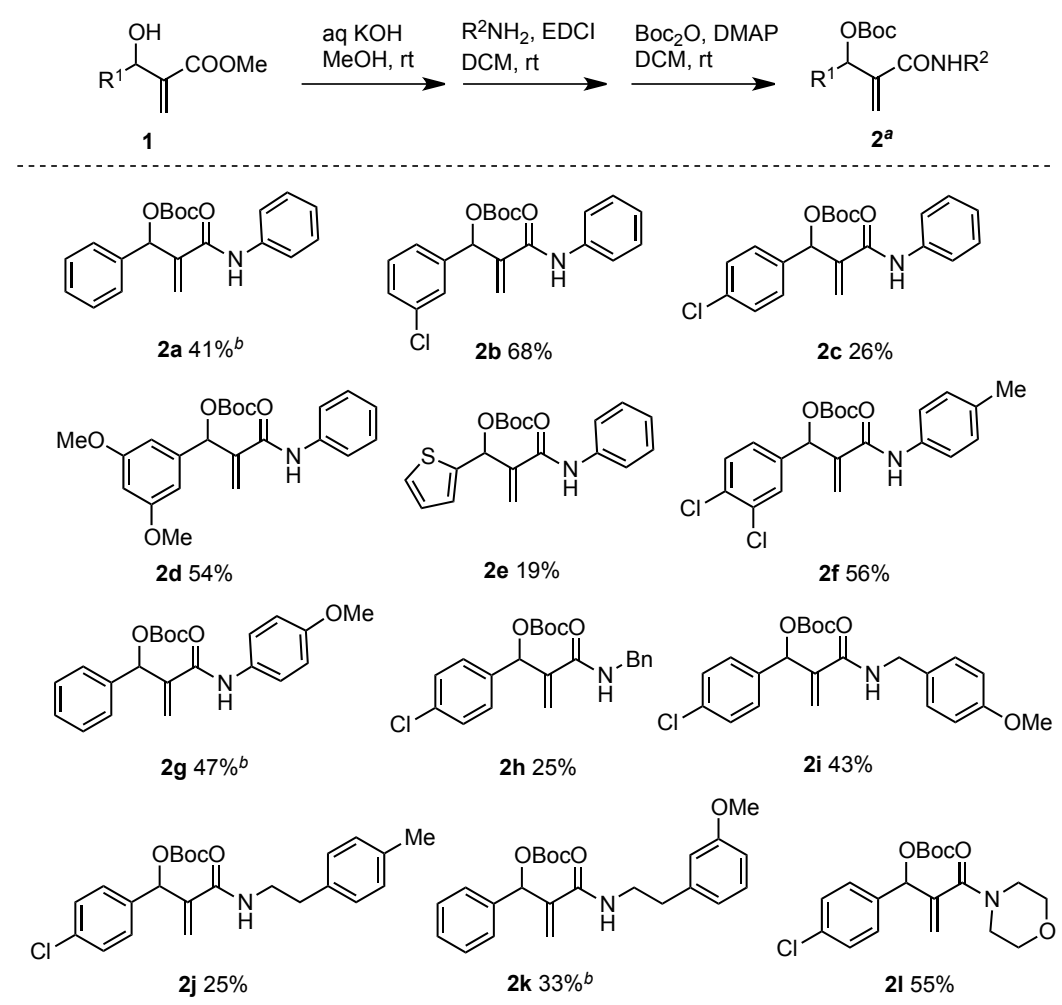

Scheme 2. Synthesis of MBH Carbonates Bearing Amide Group. ${ }^{a, b}$

$a$ Total yield for three steps.

${ }^{b}$ Toal yield for two steps using acid as starting material. 
Next, the formal [3+2] cycloaddition of tetrahydroisoquinoline 3a and $\mathrm{MBH}$ carbonates 2a was selected as model reaction. In the presence of $\mathrm{FeCl}_{3}$, the desired amide-bearing dihydropyrrolo[2,1-a]isoquinoline 4a could be detected in $20 \%$ by ${ }^{1} \mathrm{H}$ NMR. A range of iron salts and copper salts have been screened as summarized in Table 1 (entries 1-9). Among the tested iron salts, iron chloride hydrate gave the best results in terms of yield (43\% yield); while in the presence of anhydrous iron chloride, $20 \%$ yield was observed (entries 1-7). It indicates that the presence of water may play important role in the transformation. According to previous studies by $\mathrm{Li}$ and co-workers, water brought into the reaction system by the use of iron(III) chloride hexahydrate may facilitate the proton transfer.[16] It could also be the reasonable explanation for the improvement of yield in our study. The use of copper salts instead of iron chloride hydrate could not give better yield under the current conditions (entries 8-9).

Table 1. Reaction Optimization.[a-g]

\begin{tabular}{|c|c|c|c|c|c|c|}
\hline Entry & Cat & $\mathrm{mol} \%$ & Solvent & $\mathrm{T}\left({ }^{\circ} \mathrm{C}\right)$ & $t(h)$ & Yield [\%] \\
\hline 1 & $\mathrm{FeCl}_{3}$ & 54 & DMSO & 130 & 8 & $20^{[b]}$ \\
\hline 2 & $\mathrm{Fe}(\mathrm{OTf})_{3}$ & 54 & DMSO & 130 & 8 & $10^{[b]}$ \\
\hline 3 & $\mathrm{Fe}(\mathrm{OTf})_{2}$ & 54 & DMSO & 130 & 8 & $26^{[b]}$ \\
\hline 4 & $\mathrm{FeCl}_{2}$ & 54 & DMSO & 130 & 8 & $22^{[b]}$ \\
\hline 5 & $\mathrm{FeCl}_{3} \cdot 6 \mathrm{H}_{2} \mathrm{O}$ & 54 & DMSO & 130 & 8 & $43^{[b]}$ \\
\hline 6 & $\mathrm{Fe}_{2}\left(\mathrm{SO}_{4}\right)_{3}$ & 54 & DMSO & 130 & 8 & $17^{[b]}$ \\
\hline 7 & $\mathrm{FePO}_{4}$ & 54 & DMSO & 130 & 8 & $18^{[b]}$ \\
\hline 8 & $\mathrm{Cu}(\mathrm{OAc})_{2}$ & 54 & DMSO & 130 & 8 & $9^{[b]}$ \\
\hline 9 & $\mathrm{CuCl}_{2}$ & 54 & DMSO & 130 & 8 & $24^{[b]}$ \\
\hline 10 & $\mathrm{FeCl}_{3} \cdot 6 \mathrm{H}_{2} \mathrm{O}$ & 54 & DMSO & 150 & 2 & $54^{[c]}$ \\
\hline 11 & $\mathrm{FeCl}_{3} \cdot 6 \mathrm{H}_{2} \mathrm{O}$ & 20 & DMSO & 150 & 3 & $49^{[b]}$ \\
\hline 12 & $\mathrm{FeCl}_{3} \cdot 6 \mathrm{H}_{2} \mathrm{O}$ & 5 & DMSO & 150 & 6 & $33^{[c]}$ \\
\hline 13 & $\mathrm{FeCl}_{3} \cdot 6 \mathrm{H}_{2} \mathrm{O}$ & 20 & Neat & 150 & 3 & $28^{[b]}$ \\
\hline $14^{[\mathrm{d}, \mathrm{e}]}$ & $\mathrm{FeCl}_{3} \cdot 6 \mathrm{H}_{2} \mathrm{O}$ & 20 & DMSO & 150 & 2 & $51^{[\mathrm{bc}]}$ \\
\hline $15^{[f]}$ & $\mathrm{FeCl}_{3} \cdot 6 \mathrm{H}_{2} \mathrm{O}$ & 20 & DMSO & 150 & 8 & $48^{[b]}$ \\
\hline $16^{[e, g]}$ & $\mathrm{FeCl}_{3} \cdot 6 \mathrm{H}_{2} \mathrm{O}$ & 20 & DMSO & 150 & 2 & $43^{[\mathrm{cc}]}$ \\
\hline
\end{tabular}

[a] Reaction conditions: $0.15 \mathrm{mmol}$ of $3 \mathrm{a}, 0.09 \mathrm{mmol}$ of $\mathbf{2 a}$ and $0.05 \mathrm{mmol}$ of catalyst in $0.5 \mathrm{~mL}$ of solvent.

[b] The yield was determined by ${ }^{1} \mathrm{H}$ NMR using $\mathrm{CH}_{2} \mathrm{Br}_{2}$ as internal standard. 
[c] Isolated yield.

[d] With $20 \mathrm{~mol} \%$ of Bipyridine as ligand.

[e] 3a:2a = 1.5:1.

[f] 3a:2a = 1:1.6.

[g] Under $\mathrm{O}_{2}$ atmosphere.
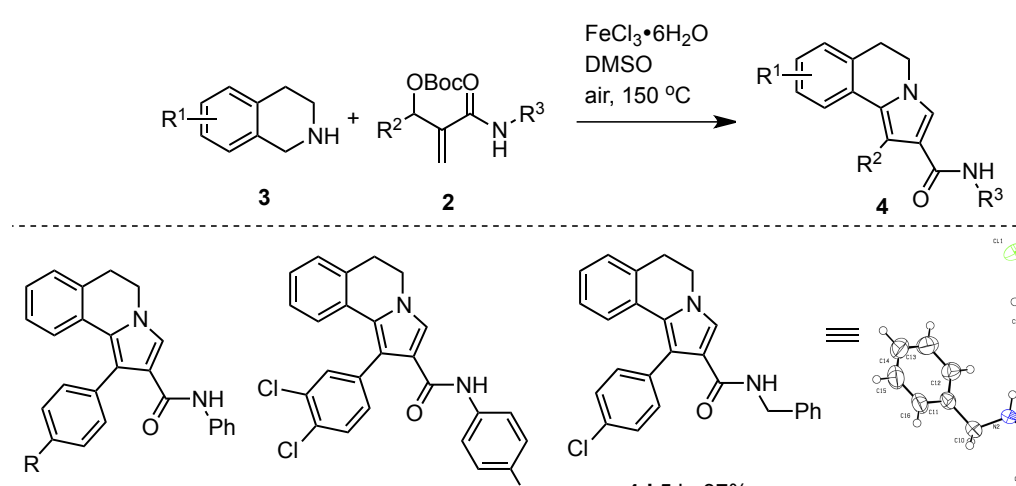

4a $\mathrm{R}=\mathrm{H}, 5 \mathrm{~h}, 42 \%$

4c $5 \mathrm{~h}, 43 \%$

4d 5 h, $37 \%$

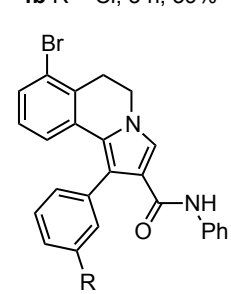

$4 \mathrm{e} R=\mathrm{H}, \quad 5 \mathrm{~h}, 62 \%$ 4f $\mathrm{R}=\mathrm{Cl}, 3.5 \mathrm{~h}, 49 \%^{\mathrm{c}}$

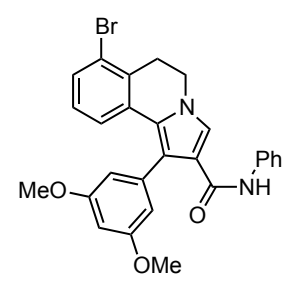

4g 5 h, 32\%d

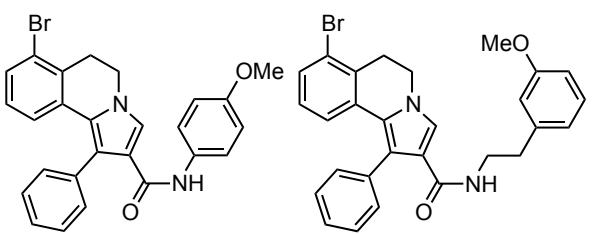

4j 5 h, 44\%

4k 5 h, 49\%

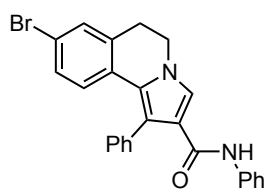

4n 4 h, 38\%

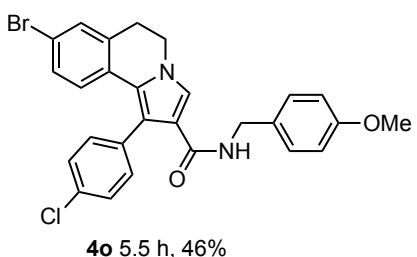

4o 5.5 h, $46 \%$

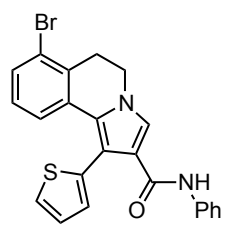

4h 5 h, $41 \%$

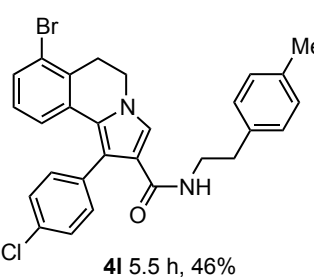

4I 5.5 h, $46 \%$

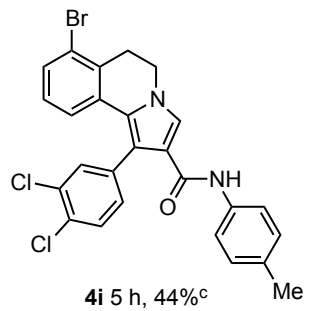<smiles>COc1cc2c(cc1OC)N1CCC2C(C(=O)Nc2ccccc2)C1</smiles>

4s 3 h, $42 \%$

Scheme 3. Substrate Scope Examination. $a, b,[18]$

${ }^{a}$ Reaction conditions: 3 (1.5 equiv), 2 (1.0 equiv) and $\mathrm{FeCl}_{3} \bullet 6 \mathrm{H}_{2} \mathrm{O}(20 \mathrm{~mol} \%)$ in DMSO (1.0 M) at $150^{\circ} \mathrm{C}$ under air atmosphere.

${ }^{b}$ Isolated yield. 
${ }^{c}$ Under $\mathrm{O}_{2}$ atmosphere.

${ }^{d}$ With $50 \mathrm{~mol} \%$ of catalyst.

Since base may be beneficial for the generation of 1,n-dipole, additional base would be helpful for this reaction. However, it was found that the addition of base (DBU, $\mathrm{Na}_{2} \mathrm{CO}_{3}, \mathrm{~K}_{3} \mathrm{PO}_{4}$, DIPEA, DMAP) could not improve the reaction yield (results not shown in the Table 1). Performing the reaction at higher temperature resulted in an improved yield (54\%, entry 10). A lower catalyst loading (20 mol\%) gave a decreased yield (49\%, entry 11). Poor reaction yield was obtained when using $5 \mathrm{~mol} \%$ of catalyst (33\%, entry 12). The yield decreased dramatically when performed in a solvent-free manner $(28 \%$, entry 13). Other solvents, such as DMF, NMP and PhCl, failed to improve the yield too.

It is reported by Pappo and co-workers that ligand may play an important role in iron-catalyzed reaction.[17] However, no significant influence was observed in our catalytic system in the presence of bipyridine as ligand (entry 14). It was found that the use of excess amount of MBH carbonate failed to improve reaction yield (entry 15). Slightly lower yield was obtained when the reaction was carried out under dioxygen atmosphere instead of air atmosphere, albeit the reaction completed in a shorter reaction time (43\%, entry 16$)$.

Then we prepared a range of dihydropyrrolo[2,1-a]isoquinolines bearing amide group under the optimal reaction conditions. ${ }^{[18]}$ As shown in Scheme 3, the use of aromatic amines derived $\mathrm{MBH}$ carbonates afforded dihydropyrrolo[2,1-a]isoquinolines (4a-4c, 4e-4j, 4n, 4p, 4q and 4s) successfully (32-62\% yield). Reactions of MBH carbonate prepared with alkylated amines proceeded smoothly gaving corresponding dihydropyrrolo[2,1-a] isoquinolines (37-49\% yields, 4d, 4k, $4 \mathbf{l}$ and 4o). Thiophene unit can be incorporated into the product (compound $\mathbf{4 h}$ ). It is worthy noted that the cyclic amine derived MBH carbonate can be employed in this reaction system leading to the formation of desired product (compound $\mathbf{4 m}$ ). Variation of substituent on tetrahydroisoquinolines was possible. Generally, electronic nature and positions of substituents have no significant influence on reaction yield. Unexpectedly, the presence of strong electron-withdrawing group resulted in the failure of preparation of compound 4r. In this case, oxidation of 7-nitro-1,2,3,4-tetrahydroisoquinoline to 7-nitroisoquinoline is a competing reaction, as 7-nitroisoquinoline could be isolated as a major product under the current reaction conditions. In general, acceptable to moderate yields were obtained in most cases. Two possible reasons should account for the relatively low yields in this study: (1) Only moderate yield of SN2' product can be formed in the reaction system as amide group in the substrate may participate $\mathrm{N}$-allylic alkylation reaction; (2) Side reactions of desired products 4, such as iron mediated/catalyzed dimerization and decomposition of products 4 via the formation of heteroarene cation radicals.[8f,19] Therefore, compounds 4 would gradually diminish if heated at $150{ }^{\circ} \mathrm{C}$ for longer reaction time (monitored by TLC). 
We further tested tetrahydro- $\beta$-carboline $\mathbf{5}$ under the optimized reaction conditions as shown in Scheme 4. Only SN2' product 6a can be isolated in 56\% yield after heating at $150{ }^{\circ} \mathrm{C}$ for $1 \mathrm{~h}$ and no significant formation of cyclized product $\mathbf{6 b}$ can be observed at this time; while only $19 \%$ yield of compound $\mathbf{6 b}$ could be obtained after heated at $150{ }^{\circ} \mathrm{C}$ for 5 h. ${ }^{[18]}$ The low yield should be due to the instability of the final product $\mathbf{6 b}$ in the reaction system because of the same reason mentioned above.
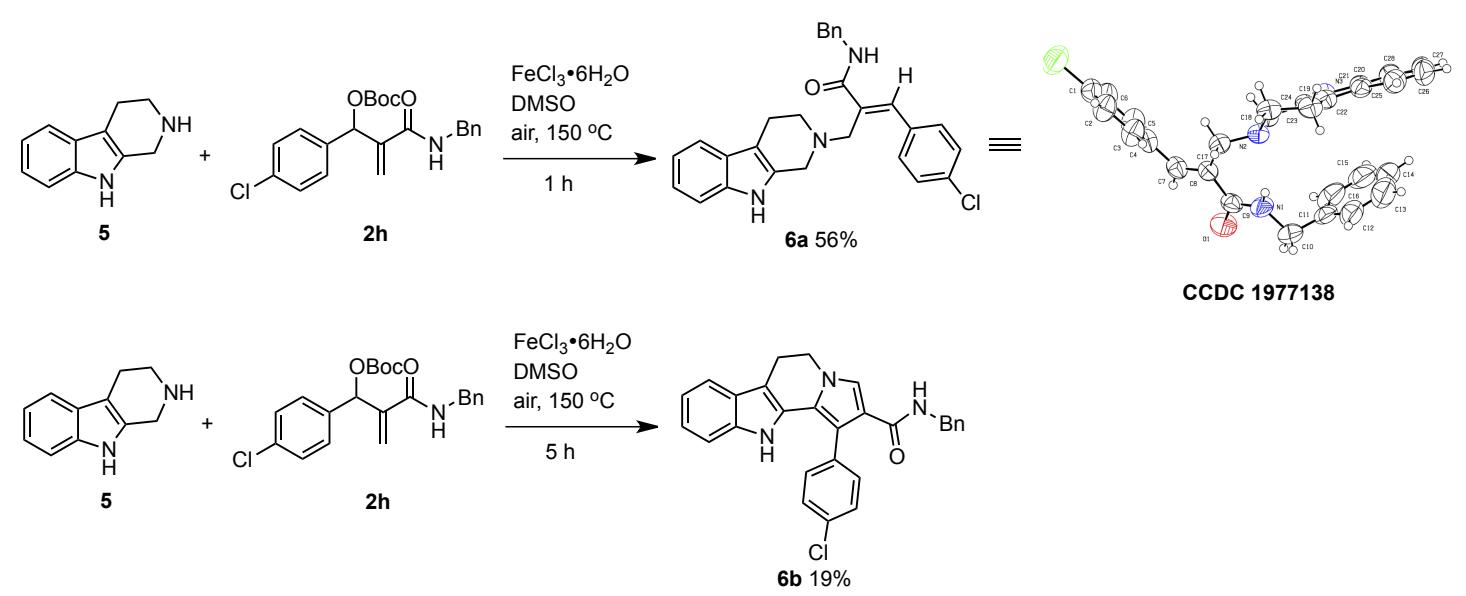

Scheme 4. Reaction of Tetrahydro- $\beta$-carboline $\mathbf{5}$ with MBH carbonate $\mathbf{2 h}$. $^{[18]}$

To demonstrate the potential application of this methodology, further transformations of dihydropyrrolo[2,1-a]isoquinolines have been carried out as shown in Scheme 5. Suzuki coupling of compound 4e afforded highly functionalized pyrrolo[2,1-a] isoquinoline 7 in $67 \%$ yield.

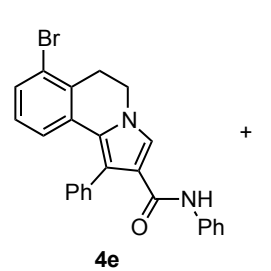

$4 \mathrm{e}$

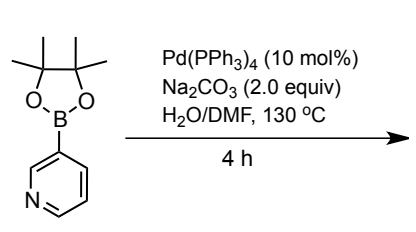

Scheme 5. Transformation.

Mechanistic study has been carried out as shown in Scheme 6. Intermediate $\mathbf{8 a}$ could be formed easily in 66\% yield by stirring the solution of $\mathbf{3 a}$ and $\mathbf{2 a}$ in DCM at rt (Scheme 6, eq 1). No dihydropyrrolo[2,1-a]isoquinoline 4a can be detected in this reaction. In the absence of catalyst, the intermediate 8a and dihydropyrrolo[2,1-a]isoquinoline 4 a could be observed in $10 \%$ and $20 \%$ NMR yields respectively after heating the reaction at $150{ }^{\circ} \mathrm{C}$ for $5 \mathrm{~h}$ (eq 2); While similar results were obtained by performing the reaction in dark $(4 \%$ and $19 \%$ NMR yield respectively, eq 3). It indicates that the reaction can be achieved by heat, while light has no influence on this reaction. Heating intermediate $\mathbf{8 a}$ in the presence of iron salts at $150{ }^{\circ} \mathrm{C}$ for $5 \mathrm{~h}$ resulted in the formation of product $\mathbf{4 a}$ in 
$40 \%$ yield (eq 4). Obviously, iron salts in this reaction can improve the reaction yield significantly and is essential for this reaction.
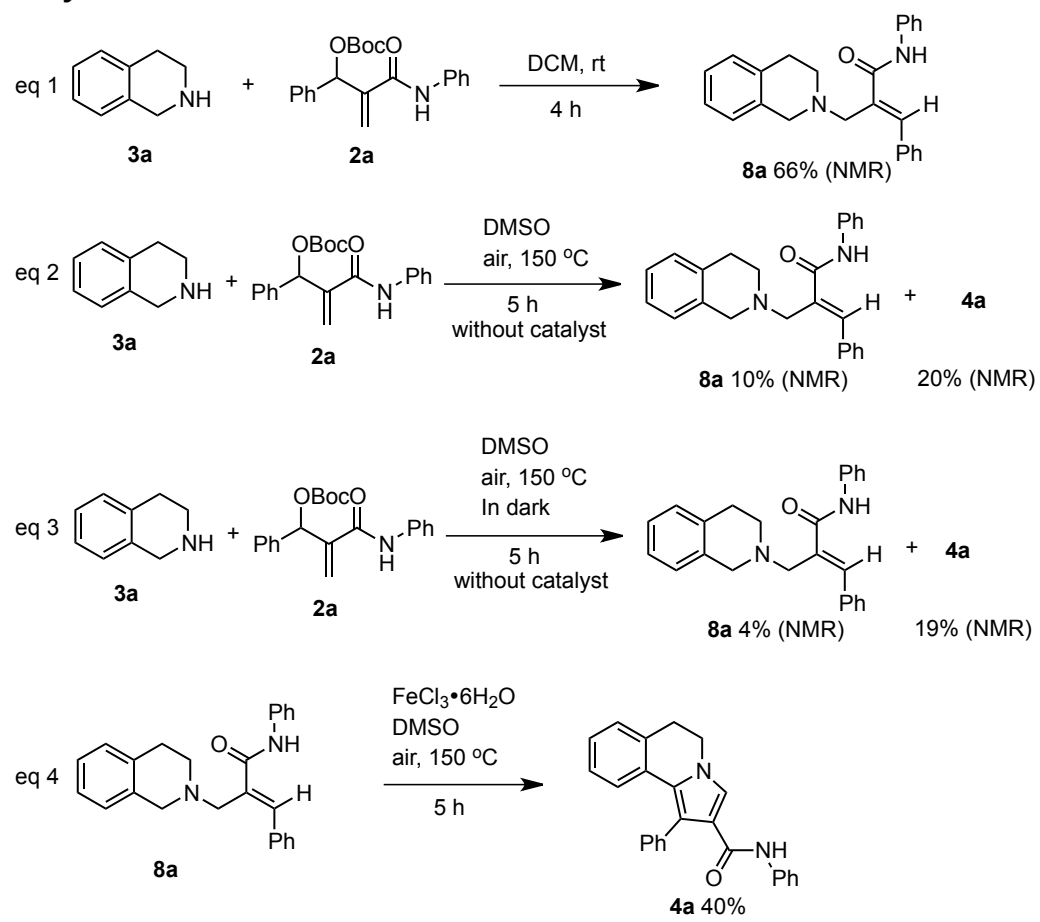

Scheme 6. Mechanistic Study.

On the basis of our results and previous studies, a plausible mechanism has been proposed as shown in Scheme 7. Firstly, the attack of cyclic amine 3a to MBH carbonate 2a yields SN2' product 8a. The 1,3-dipolar B can be formed by oxidation in the presence of iron salts and further deprotonation with the in situ generated base. Electrocyclization of $\mathbf{B}$ and $\mathbf{C}$ leads to the generation of tetrahydropyrrolo[2,1-a]isoquinoline D. A final aromatization of compound D produces dihydropyrrolo[2,1-a]isoquinoline $\mathbf{4 a}$.

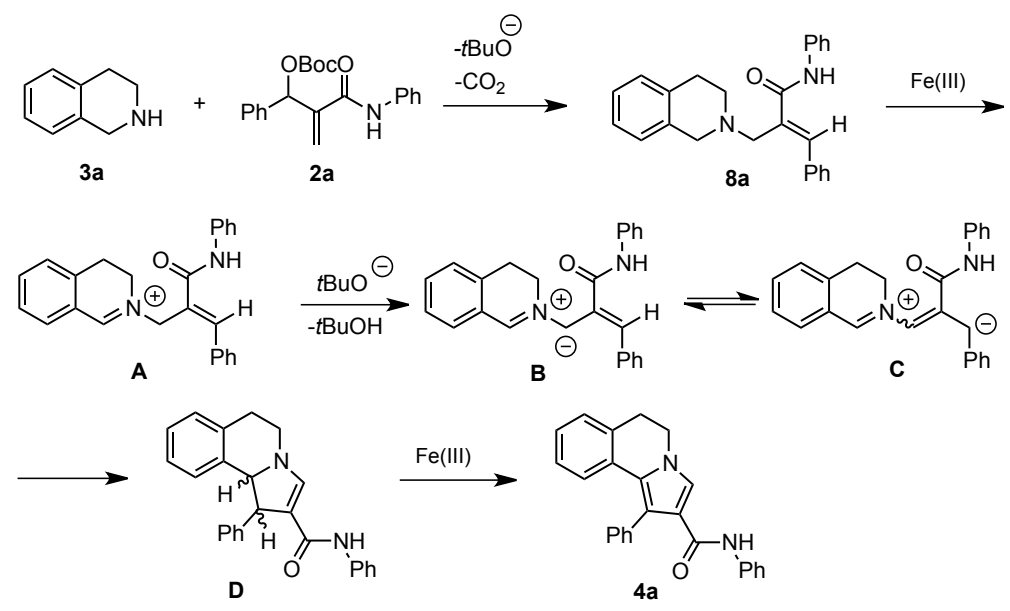

Scheme 7. Proposed Mechanism.

\section{Conclusions}


In conclusion, we have developed an iron(III) chloride hexahydrate catalyzed formal [3+2] cycloaddition of tetrahydroisoquinolines and amide-containing MBH carbonates. The efficient synthesis of amide-bearing dihydropyrrolo[2,1-a] isoquinolines could be achieved through iron catalyzed SN2'/oxidation/electrocyclization/aromatization cascade. Notably, a series of new MBH carbonates bearing amide group has been prepared by hydrolysis/ amidation/carbonation with easily accessible material.

\section{Experimental Section}

General methods ${ }^{1} \mathrm{H}$ NMR and ${ }^{13} \mathrm{C}$ NMR spectra were recorded at Bruker Avance 400. Chemical shifts are reported in ppm downfield from $\mathrm{CDCl}_{3}(\delta=7.26$ $\mathrm{ppm})$ for ${ }^{1} \mathrm{H}$ NMR and relative to the central $\mathrm{CDCl}_{3}$ resonance $(\delta=77.0 \mathrm{ppm})$ for ${ }^{13} \mathrm{C}$ NMR spectroscopy. Coupling constants are given in Hz. ESI-MS analysis was performed using a LTQ Orbitrap mass spectrometer.

All reagents and solvents were obtained from commercial sources and used without further purification unless otherwise noted. MBH carbonates 2 were prepared according to reported procedure. ${ }^{[9,15]}$

General procedure for the synthesis of compound 4: A mixture of tetrahydroisoquinoline 3 (1.5 equiv), MBH carbonate 2 (1.0 equiv) and $\mathrm{FeCl}_{3} \bullet 6 \mathrm{H}_{2} \mathrm{O}(20 \mathrm{~mol} \%)$ in DMSO $(1.0 \mathrm{M})$ was stirred at $150{ }^{\circ} \mathrm{C}$ under air atmosphere (monitored by TLC). Upon heated for indicated time shown in Scheme 3, the mixture was cooled to rt, diluted with DCM, washed with water and purified directly by a silica gel flash chromatography (Hexane/EtOAc) to afford compound 4 .

\section{Acknowledgements}

We are grateful for the support provided for this study by the National Natural Science Foundation of China $(21871035,21502013)$.

\section{Conflict of Interest}

The authors declare no conflict of interest.

Keywords: Iron chloride - Dihydropyrrolo[2,1-a]isoquinoline • Tetrahydroisoquinoline $\bullet$ Cycloaddition $\bullet$ Morita-Baylis-Hillman carbonate

\section{References:}

[1] a) H. Fan, J. Peng, M. T. Hamann, J.-F. Hu, Chem. Rev. 2008, 108, 264; b) D. Baunbæk, N. Trinkler, Y. Ferandin, O. Lozach, P. Ploypradith, S. Rucirawat, F. Ishibashi, M. Iwao, L. Meijer, Mar. Drugs. 2008, 6, 514; c) H.-N. Lv, K.-W. Zeng, M.-B. Zhao, Y. Jiang, P.-F. Tu, J Asian Nat Prod Res. 2017, 20, 195. 
[2] a) W. Lin, S. Ma, Org. Chem. Front. 2017, 4, 958; b) P. Ploypradith, C. Mahidol, P. Sahakitpichan, S. Wongbundit, S. Ruchirawat, Angew. Chem. Int. Ed. 2004, 43, 866; c) L. Shen, N. Xie, B. Yang, Y. Hu, Y. Zhang, Eur. J. Med. Chem. 2014, 85, 807; d) S. Boonya-udtayan, N. Yotapan, C. Woo, C. J. Bruns, S. Ruchirawat, N. Thasana, Chem. Asian. J. 2010, 5, 2113; e) Q. Li, J. Jiang, A. Fan, Y. Cui, Y. Jia, Org. Lett. 2011, 13, 312; f) S. T. Handy, Y. Zhang, H. Bregman, J. Org. Chem. 2004, 69, 2362.

[3] a) H.-M. Huang, Y.-J. Li, Q. Ye, W.-B. Yu, L. Han, J.-H. Jia, J.-R. Gao, J. Org. Chem. 2014, 79, 1084; b) S. Su, J. A. Porco. Jr, J. Am. Chem. Soc., 2007, 129, 7744; c) A. Fujiya, M. Tanaka, E. Yamaguchi, N. Tada, A. Itoh, J. Org. Chem. 2016, 81, 7262; d) S. Nekkanti, N. P. Kumar, P. Sharma, A. Kamal, F. M. Nachtigall, O. Forero-Doria, L. S. Santos, N. Shankaraiah, RSC Adv. 2016, 6, 2671; e) C. Vila, J. Lau, M. Rueping, Beilstein J. Org. Chem. 2014, 10, 1233; f) C. Yu, Y. Zhang, S. Zhang, H. Li, W. Wang, Chem. Commun. 2011, 47, 1036; g) R. Grigg, F. Heaney, J. Chem. Soc. Perkin Trans. 1989, 198; h) J. Krishnan, B. Vedhanarayanan, B.S. Sasidhar, S. Varughese, V. Nair, Chem. Asian J. 2017, 12, 623; h) D. Liu, J. Sun, Y. Zhang, C.-G. Yan, Org. Biomol. Chem., 2019, 17, 8008; i) K. M. Dawood, M. B. Elamin, A. M. Farag, J. Heterocyclic Chem., 2015, 53, 1928.

[4] a) M. Leonardi, M. Villacampa, J. C. Menéndez, J. Org. Chem. 2017, 82, 2570; b) R. Chen, Y. Zhao, H. Sun, Y. Shao, Y. Xu. M. Ma, L. Ma, X. Wan, J. Org. Chem. 2017, 82, 9291; c) K.-L. Zheng, M.-Q. You, W.-M. Shu, Y.-D. Wu, A.-X. Wu, Org. Lett. 2017, 19, 2262; d) K. Zheng, S. Zhuang W. Shu, Y. Wu, C. Yang, A.-X. Wu, Chem. Commun. 2018, 54, 11897; e) L. G. Voskressensky, T. N. Borisova, M. D. Matveeva, V. N. Khrustalev, A.V. Aksenov, A. A. Titov, A. E. Vartanova, A. V. Varlamov, RSC Adv. 2016, 6, 74068; f) K. Zheng, S. Zhuang, M. You, W. Shu, A. Wu, Y. Wu, Chemistryselect. 2017, 2, 10762.

[5] a) Y.-Q. Zou, L.-Q. Lu, L. Fu, N.-J. Chang, J. Rong, J.-R. Chen, W.-J. Xiao, Angew. Chem. Int. Ed. 2011, 50, 7171; b) L, Huang, J. Zhao, Chem. Commun. 2013, 49, 3751; c) S. Guo, H. Zhang, L. Huang, Z. Guo, G. Xiong, J. Zhao, Chem. Commun. 2013, 49, 8689; d) Y.-H. Jiang, J. Sun, Q. Sun, C.-G. Yan, Asian. J. Org. Chem. 2017, 6, 862.

[6] a) D. Basavaiah, B. Devendar, D. V. Lenin, T. Satyanarayana, Synlett., 2009, 411; b) R. Grigg, P. Myers, A. Somasunderam, V. Sridharan, Tetrahedron, 1992, 48, 9735; c) Z. Yang, N. Lu, Z. Wei, J. Cao, D. Liang, H. Duan, Y. Lin, J. Org. Chem., 2016, 81, 11950.

[7] a) K. B. Manjappa, J.-R. Syu, D.-Y. Yang, Org. Lett. 2016, 18, 332; b) Y. Yu, Y. Liu, A. Liu, H. Xie, H. Li, W. Wang, Org. Biomol. Chem., 2016, 14, 7455; c) C. Feng, Y. Yan, Z. Zhang, K. Xu, Z. Wang, Org. Biomol. Chem., 2014, 12, 4837; d) X.-M. Xu, L. Zhao, J. Zhu, M.-X. Wang, Angew. Chem. Int. Ed. 2016, 55, 3799; e) W. Liu, S.-T. Du, S.-Y. Wang, and W.-W. Liao, J. Org. Chem., 2017, 82, 4829; f) J. Liu, P. Chakraborty, H. Zhang, L. Zhong, Z.-X. Wang, X. Huang, ACS Catal. 2019, 9, 2610; g) T. Punirun, D. Soorukram, C. Kuhakarn, V. Reutrakul, M. Pohmakotr, J. Org. Chem. 2018, 83, 765.

[8] a) H.-L. Cui, J.-F. Wang, H.-L. Zhou, X.-L. You, X.-J. Jiang, Org. Biomol. Chem., 2017, 15, 3860; b) S.-W. Liu, Y.-J. Gao, Y. Shi, L. Zhou, X. Tang, H.-L. Cui, J. Org. Chem., 2018, 83, 13754; c) X. Tang, M.-C. Yang, C. Ye, L. Liu, H.-L. Zhou, X.-J. Jiang, X.-L. You, B. Han, H.-L. Cui, Org. Chem. Front., 2017, 4, 2128; d) X. Tang, Y.-J. Gao, H.-Q. Deng, J.-J. Lei, S.-W. Liu, L. Zhou, Y. Shi, H. Liang, J. Qiao, L. Guo, B. Han, H.-L. Cui, Org. Biomol. Chem., 2018, 16, 3362; e) S.-W. Liu, C. Yuan, X.-F. Jiang, X.-X. Wang, H.-L. Cui, Asian. J. Org. Chem. 2020, 9, 82; f) X.-F. Jiang, H. Tan, H.-L. Cui, Org. Biomol. Chem., 2020, 18, 660. 
[9] H.-L. Cui, L. Jiang, H. Tan, S. Liu, Adv. Synth. Catal. 2019, 361, 4772.

[10] a) T. M. V. D. Pinho e Melo, Eur. J. Org. Chem., 2006, 2873; b) D. Seidel, Acc. Chem. Res., 2015, 48, 317; c) X. Xu, M. P. Doyle, Acc. Chem. Res., 2014, 47, 1396; d) I, Coldham, R. Hufton, Chem. Rev., 2005, 105, 2765; e) N. De, E. J. Yoo, ACS Catal., 2018, 8, 48; f) O. Anaç, F. Ş. Güngör, Tetrahedron, 2010, 66, 5931; g) M. Nyerges, J. Tóth, P. W. Groundwater, Synlett, 2008, 9, 1269; h) N. A. Nedolya, B. A. Trofimov, Chem. Heterocycl. Com., 2013, 49, 152; i) V. Nair, A. Deepthi, D. Ashok, A. E. Raveendran, R. R. Paul, Tetrahedron, 2014, 70, 3085.

[11] D. Basavaiah, B. Lingaiah, G. C. Reddy, B. C. Sahu, Eur. J. Org. Chem. 2016, 2398.

[12] a) A. A. O. Sarhan, C. Bolm, Chem. Soc. Rev. 2009, 38, 2730; b) C. Bolm, J. Legros, L. L. Paih, L. Zani, Chem. Rev. 2004, 104, 6217; c) I. Bauer, H.-J. Knölker, Chem. Rev. 2015, 115, 3170; d) K. Gopalaiah, Chem. Rev. 2013, 113, 3248; e) J. Legros, B. Figadère, Nat. Prod. Rep. 2015, 32, 1541; f) A. Fürstner, ACS Cent. Sci. 2016, 2, 778.

[13] a) T.-Y. Liu, M. Xie, Y.-C. Chen, Chem. Soc. Rev. 2012, 41, 4101; b) P. Xie, Y. Huang, Org. Biomol. Chem. 2015, 13, 8578; c) V. Singh, S. Batra, Tetrahedron. 2008, 64, 4511; d) D. Basavaiah, B. S. Reddy, S. S. Badsara, Chem. Rev. 2010, 110, 5447; e) Y. Wei, M. Shi, Chem. Rev. 2013, 113, 6659; f) D. Basavaiah, G. Veeraraghavaiah, Chem. Soc. Rev. 2012, 41, 68.

[14] a) R. M. de Figueiredo, J.-S. Suppo, J.-M. Campagne, Chem. Rev., 2016, 116, 12029; b) D. G. Brown, J. Boström, J. Med. Chem., 2016, 59, 4443; c) C. L. Allen, J. M. J. Williams, Chem. Soc. Rev., 2011, 40, 3405; d) A. Ojeda-Porrasand, D. Gamba-Sańchez, J. Org. Chem., 2016, 81, 11548; e) J. R. Dunetz, J. Magano, G. A. Weisenburger, Org. Process Res. Dev., 2016, 20, 140.

[15] a) J. Feng, X. Lu, A. Kong, X. Han, Tetrahedron, 2007, 63, 6035; b) K. H. Kim, H. S. Lee, J. N. Kim, Tetrahedron Lett, 2009, 50, 1249.

[16] X. Guo, R. Yu, H. Li, Z. Li, J. Am. Chem. Soc. 2009, 131, 17387.

[17] R. Parnes, U. A. Kshirsagar, A. Werbeloff, C. Regev, D. Pappo, Org. Lett. 2012, 14, 3324.

[18] CCDC 1977137 (4d) and CCDC 1977138 (6a) contain the supplementary crystallographic data for this paper. These data can be obtained free of charge from The Cambridge Crystallographic Data Centre via www.ccdc.cam.ac.uk/data_request/cif.

[19] a) M. Schmittel, A. Burghart, , Angew. Chem. Int. Ed. 1997, 36, 2551; b) M. Grzybowski, K. Skonieczny, H. Butenschçn, D. T. Gryko, Angew. Chem. Int. Ed. 2013, 52, 9900; b) H.-L. Cui, Org. Biomol. Chem., 2020, DOI: 10.1039/D0OB00441C. 
Other files

191118CHL_0m.cif (470.02 KiB)

view on ChemRxiv - download file 